Y.-G. Lu

Nagoya Math. J.

Vol. 139 (1995), 173-183

\title{
QUANTUM MARKOV CHAINS AND CLASSICAL RANDOM SEQUENCES
}

\author{
YUN-GANG LU
}

\section{§1. Introduction}

Quantum Markov chain introduced by Accardi (cf. $[1,2,3])$ is one of natural generalization of classical Markov chain. It has many interesting applications in physics and the most important one is given by the paper of FannesNachtergaele-Werner ([4]), where an application of quantum Markov chain's technique enables us to understand the Valence bond states well.

In this note, by the terminology of quantum Markov chain we follow Accardi's definition (cf. [1] or [3]):

Let be given a $C^{*}$-algebra $\mathscr{B}=\mathbf{B}(H)$ for some fixed separable Hilbert space $H$ and $\mathscr{A}:=\bigotimes_{\mathrm{N}} \mathscr{B}$ (where, $\otimes$ means the $C^{*}$-tensor induced by the usual tensor product of Hilbert space). For each $n \in \mathbf{N}$, let be given a homomorphism

$$
j_{n}: \mathscr{B} \hookrightarrow 1_{0} \otimes 1_{1} \otimes \cdots \otimes 1_{n-1} \otimes \mathscr{B} \otimes 1_{n+1} \otimes 1_{n+2} \otimes \cdots=: 1_{n)} \otimes \mathscr{B} \otimes 1_{(n}
$$

such that

$$
j_{n}(\alpha):=1_{n)} \otimes a \otimes 1_{(n}, \quad \forall a \in \mathscr{B} .
$$

A bilinear map $\mathscr{E}$ from $\mathscr{B} \otimes \mathscr{B}$ to $\mathscr{B}$ is called a transition expectation if it is completely positive and identity preserving.

With above notations, quantum Markov chain (or quantum Markovian state) is defined on $\mathscr{A}:=\bigotimes_{\mathrm{N}} \mathscr{B}$ as the following: a state $\phi$ on $\mathscr{A}$ is called Markovian state if there exists a state $\phi_{0}$ (initial state) on $\mathscr{B}$ and a transition expectation $\mathscr{E}$ such that

$$
\phi\left(j_{0}\left(a_{0}\right) j_{1}\left(a_{1}\right) \cdots j_{n}\left(a_{n}\right)\right)=\phi_{0}\left(\mathscr{E}\left(a_{0} \otimes \mathscr{E}\left(a_{1} \otimes \cdots \otimes \mathscr{E}\left(a_{n-1} \otimes \mathscr{E}\left(a_{n} \otimes 1\right)\right) \cdots\right)\right) .\right.
$$

It is proved (cf. [3]) that any transition expectation $\mathscr{E}$ has the form

Received August 5, 1992.

Revised October 4, 1994. 


$$
\mathscr{E}(x)=\operatorname{Tr}_{2}\left(\sum_{j} K_{j}^{*} x K_{j}\right)
$$

where, $\operatorname{Tr}_{2}$ means the partial trace with respect to the second factor; $K_{j} \in \mathscr{B} \otimes \mathscr{B}$, $j=1,2, \cdots$. Moreover, the index $j$ runs over a finite subset of $\mathbf{N}$ if $H$ is a finite dimensional Hilbert space.

Throughout this note we restrict ourselves to the case of $\operatorname{dim}(H)=d<\infty$. Since for each orthonormal basis (o.n.b.) $\left\{e_{n}\right\}_{n=1}^{d}$ of $H, \mathbf{B}(H)$ can be considered as the algebra of all $d \times d$ matrices, i.e. $B(H) \cong M_{d}$, the further assumption is made: $\mathscr{B}=M_{d}$.

For each $K \in \mathscr{B} \otimes \mathscr{B}$, we have the expression

$$
K=\sum_{h, h^{\prime}}\left|e_{h}><e_{h^{\prime}}\right| \otimes K_{h, h^{\prime}}
$$

where $\left|e_{h}><e_{h^{\prime}}\right|$ means the matrix whose $\left(h, h^{\prime}\right)$-element is equal to 1 and elsewhere zero. In the following by the notation $\mathscr{D}$ we shall denote the diagonal subalgebra of $M_{d}$ (corresponding to $\left\{e_{h}\right\}_{h=1}^{d}$ ).

With above definitions and notations, each $\{1, \cdots, d\}$-valued classical Markov chain $\left\{X_{n}\right\}_{n=1}^{\infty}$ on a propability space $(\mathbf{P}, \Omega)$ with initial distribution $p$ and transition matrix $P=\left(p_{i, j}\right)$ can be understood as a quantum Markov chain in the following canonical way:

i) each bounded function on $\{1, \cdots, d\}$, say $f$, will be regarded as an element of $\mathscr{D}$ :

$$
\left(\begin{array}{cccc}
f(1) & 0 & \cdots & 0 \\
0 & f(2) & \cdots & 0 \\
\vdots & \vdots & \ddots & \vdots \\
0 & 0 & \cdots & f(d)
\end{array}\right)
$$

ii) for each $n \in \mathbf{N}$, let us define a homomorphism

$$
j_{n}(f):=f\left(X_{n}\right), \forall f \in \mathscr{D}
$$

iii) define

$$
K_{1, h, h}:=\left(\begin{array}{cccc}
\sqrt{p_{h, 1}} & 0 & \cdots & 0 \\
0 & \sqrt{p_{h, 2}} & \cdots & 0 \\
\vdots & \vdots & \ddots & \vdots \\
0 & 0 & \cdots & \sqrt{p_{h, d}}
\end{array}\right)
$$

$$
\begin{array}{ll}
K_{1, h, h^{\prime}}:=0, & \text { if } h \neq h^{\prime} \\
K_{n, h, h^{\prime}}:=0, \quad \forall n \geq 2
\end{array}
$$


iv) define initial state $\phi_{0}$ by

$$
\phi_{0}(\cdot):=\operatorname{Tr}\left(\left(\begin{array}{cccc}
p_{1} & 0 & \cdots & 0 \\
0 & p_{2} & \cdots & 0 \\
\vdots & \vdots & \ddots & \vdots \\
0 & 0 & \cdots & p_{d}
\end{array}\right) \cdot\right)
$$

It is easy to check that

$$
\mathscr{E}_{1}(x):=\operatorname{Tr}_{2}\left(K_{1}^{*} \cdot K_{1}\right)
$$

where $K_{1}:=\sum_{h}\left|e_{h}><e_{h}\right| \otimes K_{1, h, h}$, is a transition expectation with the property:

$$
\mathscr{E}_{1}(f \otimes g)=f \cdot \mathscr{E}_{1}(1 \otimes g) \quad \forall f, g \in \mathscr{D}
$$

and $\phi_{0}$ is a state on $M_{d}$. Therefore $\left(\phi_{0}, \mathscr{E}_{1}\right)$ is a quantum Markov chain and moreover the restriction of this quantum Markov chain to the diagonal subalgebra $\mathscr{D}$ is nothing but the given classical Markov chain.

From above discussion one can see that the choice of $\left(\phi_{0}, \mathscr{E}\right)$ is not unique. In fact we can replace the initial density matrix

$$
\left(\begin{array}{cccc}
p_{1} & 0 & \cdots & 0 \\
0 & p_{2} & \cdots & 0 \\
\vdots & \vdots & \ddots & \vdots \\
0 & 0 & \cdots & p_{d}
\end{array}\right)
$$

by any density matrix $\omega_{0}$ which has diagonal elements $\left\{p_{1}, p_{2}, \cdots, p_{d}\right\}$. Of course there are many such density matrices. Moreover we can replace $K_{1, h, h}$ by any $K_{h, h}$ which has only to possess the property:

$$
K_{h, h} K_{h, h}^{*}(j, j)=p_{h, j}, \quad \forall j=1,2, \cdots, d
$$

Let denote

$$
K_{0}:=\sum_{h}\left|e_{h}><e_{h}\right| \otimes K_{h, h}
$$

and define

$$
\mathscr{E}_{0}(\cdot):=\operatorname{Tr}_{2}\left(K_{0}^{*} \cdot K_{0}\right)
$$

Then $\left(\operatorname{Tr}\left(\omega_{0} \cdot\right), \mathscr{E}_{0}(\cdot)\right)$ is also a quantum Markov chain obtained from the same classical Markov chain $\left(p, P=\left(p_{i, j}\right)\right)$ and moreover, by restricting the two quantum Markov chains $\left(\operatorname{Tr}\left(\omega_{0} \cdot\right), \mathscr{E}_{0}(\cdot)\right)$ and $\left(\phi_{0}, \mathscr{E}_{1}\right)$ on $\mathscr{D}$, one obtains the same classical Markov chain $\left\{p, P=\left(p_{i, j}\right)\right)$. 
Now a natural problem can be asked: starting from a classical Markov chain $\left\{p, P=\left(p_{i, j}\right)\right)$, what is the most general form of the quantum form of the quantum Markov chains whose restriction to the diagonal subalgebra gives the classical Markov chain? One can also ask the problem reversely: which kind of quantum Markov chain gives, by restricting it to diagonal subalgebra, a classical Markov chain?

Moreover, it is usually believed that some non-Markovian stochastic processes (classical) are quantum Markov chain. Then a natural question is this: which kind of classical stochastic processes can be a quantum Markov chain? In order to answer this question, first of all we have to understand which kind of quantum Markov chain, by restricting it to diagonal subalgebra $\mathscr{D}$, gives a classical stochastic process in the sense of $\mathscr{E}$ maps $\mathscr{D} \otimes \mathscr{D}$ to $\mathscr{D}$ ?

Remark. Notice that if

$$
\mathscr{E}(\mathscr{D} \otimes \mathscr{D}) \subset \mathscr{D}
$$

then we can consider the initial density matrix $\omega_{0}$ as a diagonal one since its non-diagonal elements don't play any rule in this case.

In this note we give the answer of above questions. Our main results are stated in the next section.

\section{§2. The main results}

In the section 1 , we opened the problems and gave their motivations. Now we shall state the answer of the problems. The proof of our main results is the contents of the next section.

Proposition (2.1). A transition expectation $\mathscr{E}$ maps $\mathscr{D} \otimes \mathscr{D}$ to $\mathscr{D}$ if and only if for each $r, r^{\prime}, h \in\{1, \cdots, d\}$ with $r \neq r^{\prime}$, the matrix $\sum_{j} K_{j, h, r} K_{j, h, r^{\prime}}^{*}$ has only zero diagonal elements.

Definition (2.2). We say that an $\{1, \cdots, d\}$-valued classical stochastic process $\left\{X_{n}\right\}_{n=0}^{\infty}$ on probability space $(\mathbf{P}, \Omega)$ is the quantum Markov chain $\left(\phi_{0}, \mathscr{E}\right)$ if $\mathscr{E}$ satisfies (1.12) and the joint distributions are the same, i.e. for each $n \in \mathbf{N}$, $\left\{i_{h}\right\}_{h=0}^{n} \subset\{1, \cdots, d\}$

$$
\mathbf{P}\left(X_{0}=i_{0}, X_{1}=i_{1}, \cdots, X_{n}=i_{n}\right)
$$




$$
\begin{aligned}
& =\phi\left(\left|e_{i_{0}}><e_{i_{0}}\right| \otimes\left|e_{i_{1}}><e_{i_{1}}\right| \otimes \cdots \otimes\left|e_{i_{n}}><e_{i_{n}}\right|\right) \\
& =\phi_{0}\left(\mathscr { E } \left(| e _ { i _ { 0 } } > < e _ { i _ { 0 } } | \otimes \mathscr { E } \left(\left|e_{i_{1}}><e_{i_{1}}\right| \otimes\right.\right.\right. \\
& \left.\left.\quad \cdots \otimes \mathscr{E}\left(\left|e_{i_{n-1}}><e_{i_{n-1}}\right| \otimes \mathscr{E}\left(\left|e_{i_{n}}><e_{i_{n}}\right| \otimes 1\right)\right) \cdots\right)\right) .
\end{aligned}
$$

Now we can state the results which replies the question: which kind of classical stochastic process can be a quantum Markov chain?

THEOREM (2.3). Let $\left\{X_{n}\right\}_{n=1}^{\infty}$ be an $\{1, \cdots, d\}$-valued classical stochastic process on a probability space $(\mathbf{P}, \Omega)$. Then it is a quantum Markov chain if and only if there exist a probability measure $p_{0}$ on $\{1, \cdots, d\}$ and a 3 -indices cubic matrix $\left(T_{j, i, k}\right)$ with the following properties:

$$
\begin{gathered}
T_{j, i, k} \geq 0, \forall j, i, k \in\{1, \cdots, d\} \\
\sum_{i, k} T_{j, i, k}=1, \forall j \in\{1, \cdots, d\} \\
\mathbf{P}\left(X_{0}=j\right)=\sum_{i, k} p_{0}(i) T_{j, i, k}, \quad \forall j \in\{1, \cdots, d\}
\end{gathered}
$$

such that the joint distribution is given by:

$$
\begin{gathered}
\mathbf{P}\left(X_{0}=i_{0}, X_{1}=i_{1}, \cdots, X_{n}=i_{n}\right) \\
=\sum_{j, j_{0}, j_{1}, \cdots, j_{n}} p_{0}(j) T_{j, i_{0}, j_{0}} T_{j_{0}, i_{1}, j_{1}} \cdots T_{j_{n-2}, i_{n-1}, j_{n-1}} T_{j_{n-1}, i_{n}, j_{n}}
\end{gathered}
$$

THEOREM (2.4). Let $\left\{X_{n}\right\}_{n=0}^{\infty}$ be an $\{1, \cdots, d\}$-valued classical stochastic process on a probability space $(\mathbf{P}, \Omega)$. Then it is a quantum Markov chain if and only if there exists an $\{1, \cdots, d\}^{2}$-valued classical Markov chain $\left\{\left(Z_{n}, Y_{n}\right)\right\}_{n=1}^{\infty}$ on a probability space $\left(\mathbf{P}^{\prime}, \Omega^{\prime}\right)$ with the properties:

i) the transition probability

$$
p_{(j, i),\left(j^{\prime}, i^{\prime}\right)}:=\mathbf{P}^{\prime}\left(\left(Z_{n}, Y_{n}\right)=\left(j^{\prime}, i^{\prime}\right) \mid\left(Z_{n-1}, Y_{n-1}\right)=(j, i)\right)
$$

is independent of $i$;

ii) there exists a probability distribution $p_{0}$ on $\{1, \cdots, d\}$ and the initial distribution of $\left\{\left(Z_{n}, Y_{n}\right)\right\}_{n=0}^{\infty}$ is given by

$$
\mathbf{P}^{\prime}\left(\left(Z_{0}, Y_{0}\right)=(j, i)\right)=\sum_{k} p_{0}(k) T_{k, i, j}
$$

(where $\left(T_{i, j, k}\right)$ is determined by the process $\left\{X_{n}\right\}_{n=0}^{\infty}$ as stated in Theorem (2.3)) such that

(2.4) $\mathbf{P}\left(X_{0}=i_{0}, X_{1}=i_{1}, \cdots, X_{n}=i_{n}\right)=\mathbf{P}^{\prime}\left(Y_{0}=i_{0}, Y_{1}=i_{1}, \cdots, Y_{n}=i_{n}\right)$. 
Corollary. If the quantum Markov chain $\left(\phi_{0}, \mathscr{E}\right)$ on $\bigotimes_{\mathbf{N}} M_{d}$ has the property that $\mathscr{E}$ maps $\mathscr{D} \otimes \mathscr{D}$ to $\mathscr{D}$. Then the classical stochastic process, obtained by restricting $\left(\phi_{0}, \mathscr{E}\right)$ to the diagonal subalgebra $\mathscr{D}$, is stochastically equivalent to the second component of an $\{1, \cdots, d\}^{2}$-valued classical Markov chain $\left\{\left(Z_{n}, Y_{n}\right)\right\}_{n=0}^{\infty}$ which has the properties i), ii) in Theorem (2.4).

This Corollary is a direct conclusion of Theorems (2.3) and (2.4).

Definition (2.5). We say that the quantum Markov chain $\left(\phi_{0}, \mathscr{E}\right)$ is a classical Markov chain on the diagonal subalgebra $\mathscr{D}$ if (1.12) is valid and

$$
\mathscr{E}(\alpha \otimes b)=a \cdot \mathscr{E}(1 \otimes b), \quad \forall a, b \in \mathscr{D} .
$$

THEOREM (2.6). The quantum Markov chain $\left(\phi_{0}, \mathscr{E}\right)$ is a classical Markov chain on the diagonal subalgebra if and only if the operators $\left\{K_{j}\right\}$ (see (1.2)) have the form:

$$
K_{j}=\sum_{h}\left|e_{h}><e_{h}\right| \otimes K_{j, h, h}, \quad \forall j=1, \cdots, d
$$

Remark. The formula (2.6) means that each $K_{j}$ is a block-diagonal (not necessarily diagonal) $d^{2} \times d^{2}$ matrix, i.e.

$$
\left(\begin{array}{cccc}
K_{j, 1,1} & 0 & \cdots & 0 \\
0 & K_{j, 2,2} & \cdots & 0 \\
\vdots & \vdots & \ddots & \vdots \\
0 & 0 & \cdots & K_{j, d, d}
\end{array}\right)
$$

where, each $K_{j, l, l}(l=1, \cdots, d)$ is a $d \times d$ matrix.

\section{§3. The proof of the main results}

This section is devoted to prove our results stated in the preceding section. The basic step is to make transition expectation in more clear form. From (1.3), one knows that for each $b \in M_{d}$,

$$
\begin{aligned}
& =\sum_{j} \sum_{h, h^{\prime}, r, r^{\prime}} \operatorname{Tr}_{2}\left(\left(\left|e_{h^{\prime}}><e_{r^{\prime}}\right|^{*} \otimes K_{j, h^{\prime}, r^{\prime}}^{*}\right)\left(\left|e_{i}><e_{m}\right| \otimes b\right)\left(\left|e_{h}><e_{r}\right| \otimes K_{j, h, r}\right)\right) \\
& =\sum_{h, h^{\prime}, r, r^{\prime}}\left|e_{r^{\prime}}><e_{h^{\prime}}\right| \cdot\left|e_{i}><e_{m}\right| \cdot\left|e_{h}><e_{r}\right| \operatorname{Tr}\left(\sum_{j} K_{j, h^{\prime}, r^{\prime}}^{*} b K_{j, h, r}\right) \\
& =\sum_{r, r^{\prime}}\left|e_{r^{\prime}}><e_{r}\right| \operatorname{Tr}\left(\sum_{j} K_{j, i, r^{\prime}}^{*} b K_{j, m, r}\right) \text {. }
\end{aligned}
$$


Since $\left\{\left|e_{i}><e_{m}\right| \in M_{d}: i, m \in\{1, \cdots, d\}\right\}$ is a basis of $M_{d}$, we find that for any $a=(a(i, m)) \in M_{d}$,

$$
\mathscr{E}(a \otimes b)=\sum_{i, m} a(i, m)\left[\sum_{r, r^{\prime}}\left|e_{r^{\prime}}><e_{r}\right| \operatorname{Tr}\left(\sum_{j} K_{j, i, r^{\prime}}^{*} b K_{j, m, r}\right)\right] .
$$

By (3.2) and the fact that $\mathscr{E}$ is identity preserving, one obtains that

$$
\operatorname{Tr}\left(\sum_{j} K_{j, i, r^{\prime}}^{*} b K_{j, i, r}\right)=\delta_{r, r^{\prime}}, \quad \forall i \in \mathbf{N} .
$$

Proof of Proposition (2.1). For any $\left|e_{i}><e_{m}\right|,\left|e_{i^{\prime}}><e_{m^{\prime}}\right|$, (3.1) shows that

$$
\mathscr{E}\left(\left|e_{i}><e_{m}\right| \otimes\left|e_{i^{\prime}}><e_{m^{\prime}}\right|\right)=\sum_{r, r^{\prime}}\left|e_{r^{\prime}}><e_{r}\right| \operatorname{Tr}\left(\sum_{j} K_{j, i, r^{\prime}}^{*} \cdot\left|e_{i^{\prime}}><e_{m^{\prime}}\right| K_{j, m, r}\right) .
$$

If $\mathscr{E}$ maps $\mathscr{D} \otimes \mathscr{D}$ to $\mathscr{D}$, then for any $m, i$

$$
\begin{gathered}
\mathscr{E}\left(\left|e_{m}><e_{m}\right| \otimes\left|e_{i}><e_{i}\right|\right)=\sum_{r, r^{\prime}}\left|e_{r^{\prime}}><e_{r}\right| \operatorname{Tr}\left(\sum_{j} K_{j, m, r^{\prime}}^{*} \cdot\left|e_{i}><e_{i}\right| \cdot K_{j, m, r}\right)= \\
=\sum_{r}\left|e_{r}><e_{r}\right| \operatorname{Tr}\left(\sum_{j} K_{j, m, r} K_{j, m, r}^{*}\left|e_{i}><e_{i}\right|\right) .
\end{gathered}
$$

Therefore for any $r \neq r^{\prime}$

$$
\operatorname{Tr}\left(\sum_{j} K_{j, m, r} K_{j, m, r^{\prime}}^{*} \cdot\left|e_{i}><e_{i}\right|\right)=\sum_{j} K_{j, m, r} K_{j, m, r^{\prime}}^{*}(i, i)=0 .
$$

This shows that $\mathscr{E}$ maps $\mathscr{D} \otimes \mathscr{D}$ to $\mathscr{D}$ only if for any $m, r \neq r^{\prime}$, the matrix $\sum_{j} K_{j, m, r} K_{,, m, r^{\prime}}^{*}$ has only zero diagonal elements.

On the other hand, if for any $m, r \neq r^{\prime}$, the matrix $\sum_{j} K_{j, m, r} K_{j, m, r^{\prime}}^{*}$ has only zero diagonal elements, (3.4), (3.5) and (3.6) guarantee that $\mathscr{E}\left(\left|e_{m}><e_{m}\right| \otimes b\right)$ $\in \mathscr{D}$ for each $b \in \mathscr{D}$. Since $\left\{\left|e_{m}><e_{m}\right|\right\}_{m \in \mathbf{N}}$ is a basis of $\mathscr{D}$, it is obvious that $\mathscr{E}(a \otimes b) \in \mathscr{D}$ for each $a, b \in \mathscr{D}$. These arguments complete the proof.

Proof of Theorem (2.3). If classical stochastic process $\left\{X_{n}\right\}_{n=0}^{\infty}$ on a probability space $(\mathbf{P}, \Omega)$ is a quantum Markov chain, then by definition, there exists a pair $\left(\phi_{0}, \mathscr{E}\right)$ such that $(1.12)$ and (2.1) are valid.

First of all, let see the initial distribution. By (1.3) and (2.1), for any $i_{0} \in$ $\{1, \cdots, d\}$,

$$
\mathbf{P}\left(X_{0}=i_{0}\right)=\phi_{0}\left(\mathscr{E}\left(\left|e_{i_{0}}><e_{i_{0}}\right| \otimes 1\right)=\phi_{0}\left(\sum_{r, r^{\prime}}\left|e_{r^{\prime}}><e_{r}\right|\right) \cdot \sum_{j} \operatorname{Tr}\left(K_{j, i_{0}, r} K_{j, i_{0}, r^{\prime}}^{*}\right)\right.
$$

By (1.12), we know that (3.7) is equal to 


$$
\phi_{0}\left(\sum_{r}\left|e_{r}><e_{r}\right|\right) \cdot \sum_{j} \operatorname{Tr}\left(K_{j, i_{0}, r} K_{j, i_{0}, r}^{*}\right)
$$

Denote

$$
\phi_{0}(\cdot):=\operatorname{Tr}\left(\omega_{0} \cdot\right)
$$

with the density matrix $\omega_{0}=\left(\omega_{k, j}\right),(3.8)$ becomes

$$
\sum_{r} \omega_{r, r} \cdot \sum_{j} \operatorname{Tr}\left(K_{j, i_{0}, r} K_{j, i_{0}, r}^{*}\right)=\sum_{r} \omega_{r, r} \cdot \sum_{j} \sum_{k}\left(K_{j, i_{0}, r} K_{j, i_{0}, r}^{*}\right)(k, k) .
$$

Define

$$
p_{0}(r):=\omega_{r, r}, \quad \forall r \in\{1, \cdots, d\}
$$

and

$$
T_{r, i, k}:=\sum_{j}\left(K_{j, i, r} K_{j, i, r}^{*}\right)(k, k), \quad \forall r, i, k \in\{1, \cdots, d\} .
$$

Then $p_{0}$ is a probability measure on $\{1, \cdots, d\}$ and $(2.2 \mathrm{a}, \mathrm{b}, \mathrm{c})$ are satisfied.

Our second step is to compute the joint distribution. In order to do this we must know the explicit form of the matrix

$$
\begin{aligned}
\mathscr{E}\left(| e _ { i _ { 0 } } > < e _ { i _ { 0 } } | \otimes \mathscr { E } \left(\left|e_{i_{1}}><e_{i_{1}}\right| \otimes\right.\right. \\
\left.\left.\cdots \otimes \mathscr{E}\left(\left|e_{i_{n-1}}><e_{i_{n-1}}\right| \otimes \mathscr{E}\left(\left|e_{i_{n}}><e_{i_{n}}\right| \otimes 1\right)\right) \cdots\right)\right) .
\end{aligned}
$$

By the formulae (3.1) and (1.12), we find that (3.12) is equal to

$$
\begin{gathered}
\sum_{r_{0}}\left|e_{r_{0}}><e_{r_{0}}\right| \sum_{j_{0}} \operatorname{Tr}\left(K _ { j _ { 0 } , i _ { 0 } , r _ { 0 } } K _ { j _ { 0 } , i _ { 0 } , r _ { 0 } } ^ { * } \mathscr { E } \left(| e _ { i _ { 1 } } > < e _ { i _ { 1 } } | \otimes \cdots \otimes \mathscr { E } \left(\left|e_{i_{n-1}}><e_{i_{n-1}}\right|\right.\right.\right. \\
\left.\left.\left.\otimes \mathscr{E}\left(\left|e_{i_{n}}><e_{i_{n}}\right| \otimes 1\right)\right) \cdots\right)\right) .
\end{gathered}
$$

Applying again the formulae (3.1) and (1.12), we are able to rewrite (3.13) as

$$
\sum_{r_{0}}\left|e_{r_{0}}><e_{r_{0}}\right| \sum_{j_{0}} \operatorname{Tr}\left(K _ { j _ { 0 } , i _ { 0 } , r _ { 0 } } ^ { * 2 } \sum _ { r _ { 1 } } | e _ { r _ { 1 } } > < e _ { r _ { 1 } } | \sum _ { j _ { 0 } } \operatorname { T r } \left(K_{j_{1}, i_{1}, r_{1}}^{* 2}\right.\right.
$$

$$
\begin{gathered}
\left.\mathscr{E}\left(\left|e_{i_{1}}><e_{i_{1}}\right| \otimes \cdots \otimes \mathscr{E}\left(\left|e_{i_{n-1}}><e_{i_{n-1}}\right| \otimes \mathscr{E}\left(\left|e_{i_{n}}><e_{i_{n}}\right| \otimes 1\right)\right) \cdots\right)\right) \\
\quad=\sum_{r_{0}, r_{1}}\left|e_{r_{0}}><e_{r_{0}}\right| \sum_{j_{0}} \operatorname{Tr}\left(K _ { j _ { 0 } , i _ { 0 } , r _ { 0 } } ^ { * 2 } ( r _ { 1 } , r _ { 1 } ) \sum _ { j _ { 1 } } \operatorname { T r } \left(K_{j_{1}, i_{1}, r_{1}}^{* 2}\right.\right. \\
\left.\mathscr{E}\left(\left|e_{i_{1}}><e_{i_{1}}\right| \otimes \cdots \otimes \mathscr{E}\left(\left|e_{i_{n-1}}><e_{i_{n-1}}\right| \otimes \mathscr{E}\left(\left|e_{i_{n}}><e_{i_{n}}\right| \otimes 1\right)\right) \cdots\right)\right) .
\end{gathered}
$$

By using the symbol introduced in (3.11b), the right hand side of (3.13a) becomes

$$
\begin{gathered}
\sum_{r_{0}, r_{1}}\left|e_{r_{0}}><e_{r_{0}}\right| T_{r_{0}, i_{0}, r_{1}} \sum_{j_{1}} \operatorname{Tr}\left(K _ { j _ { 1 } , i _ { 1 } , r _ { 1 } } ^ { * 2 } \mathscr { E } \left(\left|e_{i_{1}}><e_{i_{1}}\right| \otimes\right.\right. \\
\left.\left.\cdots \otimes \mathscr{E}\left(\left|e_{i_{n-1}}><e_{i_{n-1}}\right| \otimes \mathscr{E}\left(\left|e_{i_{n}}><e_{i_{n}}\right| \otimes 1\right)\right) \cdots\right)\right) .
\end{gathered}
$$


Repeating above discussion, we find that (3.13) is equal to

$$
\sum_{r_{0}, r_{1}, \cdots, r_{n}, r}\left|e_{r_{0}}><e_{r_{0}}\right| T_{r_{0}, i_{0}, r_{1}} T_{r_{1}, r_{1}, r_{2}} \cdots T_{r_{n-1}, i_{n-1}, r_{n}} T_{r_{n}, i_{n}, r}
$$

Introducing new indices:

$$
j:=r_{0}, j_{0}:=r_{1}, \cdots, j_{n-1}:=r_{n}, j_{n}:=r
$$

(3.14) can be rewrited as

$$
\sum_{j, j_{0}, j_{1}, \cdots, j_{n}}\left|e_{j}><e_{j}\right| T_{j, i_{0}, j_{0}} T_{j_{0}, i_{1}, j_{1}} \cdots T_{j_{n-2}, i_{n-1}, j_{n-1}} T_{j_{n-1}, i_{n}, j_{n}} .
$$

By acting the initial state $\phi_{0}(\cdot)=\operatorname{Tr}\left(\omega_{0} \cdot\right)$ to $(3.14 \mathrm{a})$, one obtains $(2.2 \mathrm{~d})$ with $p_{0}(j):=\omega_{0}(j, j)$.

Now let be given a classical stochastic process $\left\{X_{n}\right\}_{n=1}^{\infty}$ with the properties (2.2a,b,c,d), we have to construct a quantum Markov chain $\left\{\phi_{0}, \mathscr{E}\right\}$ such that (1.12) and (2.1) are satisfied.

Taking the initial density matrix $\omega_{0}$ as

$$
\left(\begin{array}{cccc}
p_{0}(1) & 0 & \cdots & 0 \\
0 & p_{0}(2) & \cdots & 0 \\
\vdots & \vdots & \ddots & \vdots \\
0 & 0 & \cdots & p_{0}(d)
\end{array}\right)
$$

$\phi_{0}(\cdot):=\operatorname{Tr}\left(\omega_{0} \cdot\right)$ is, clearly, a state on $M_{d}$.

Define

$$
\begin{gathered}
K:=\sum_{h, r}\left|e_{h}><e_{r}\right| \otimes K_{h, r} \\
K_{i, j}^{*}:=\left(\sqrt{T_{j, i, 1}} e_{j}, \sqrt{T_{j, i, 2}} e_{j}, \cdots, \sqrt{T_{j, l, d}} e_{j}\right) .
\end{gathered}
$$

We have

$$
K_{i, j} K_{i, j^{\prime}}^{*}=0, \quad \forall i, j \neq j^{\prime} \in\{1, \cdots, d\}
$$

and

$$
K_{i, j} K_{i, j}^{*}(k, k)=T_{j, i, k}, \quad \forall i, j, k \in\{1, \cdots, d\} .
$$

Thus, a quantum Markov chain $\left(\phi_{0}, \mathscr{E}\right)$ is obtained and these end the proof of Theorem (2.3).

Proof of Theorem (2.4). Let classical stochastic process $\left\{X_{n}\right\}_{n=0}^{\infty}$ on a probability space $(\mathbf{P}, \Omega)$ be a quantum Markov chain. Then by Theorem (2.3), we know 
that $p_{0},\left(T_{j, i, k}\right)$ satisfy $(2.2 \mathrm{a}, \mathrm{b}, \mathrm{c}, \mathrm{d})$.

Define a probability measure $p(\cdot, \cdot)$ on $\{1, \cdots, d\}^{2}$ by

$$
p(j, i):=\sum_{r} p_{0}(r) T_{r, i, j}
$$

and a transition matrix $\left(p_{\left.(j, i), j^{\prime}, i^{\prime}\right)}\right)$ by

$$
p_{(j, i),\left(j^{\prime}, i^{\prime}\right)}:=T_{j, i^{\prime}, j^{\prime}}, \quad \forall j, i, j^{\prime}, i^{\prime} \in\{1, \cdots, d\} .
$$

Then with a canonical way we can construct a probability space $\left(\mathbf{P}^{\prime}, \Omega^{\prime}\right)$ and an $\{1, \cdots, d\}^{2}$-valued Markov chain $\left\{\left(Z_{n}, Y_{n}\right)\right\}_{n=1}^{\infty}$ with the initial distribution:

$$
\mathbf{P}^{\prime}\left(\left(Z_{0}, Y_{0}\right)=(j, i)\right)=p(j, i)=\sum_{k} p_{0}(k) T_{k, i, j}
$$

and the transition probability:

$$
\mathbf{P}^{\prime}\left(\left(Z_{n}, Y_{n}\right)=\left(j^{\prime}, i^{\prime}\right) \mid\left(Z_{n-1}, Y_{n-1}\right)=(j, i)\right)=p_{(j, i),\left(j^{\prime}, i^{\prime}\right)}=T_{j, i^{\prime}, j^{\prime}}
$$

Thus by a simple computation we know that the joint distribution $\mathbf{P}^{\prime}\left(Y_{0}=i_{0}, \cdots\right.$, $\left.Y_{1}=i_{1}\right)$ is given by the right hand side of $(2.2 \mathrm{~d})$ and this implies (2.4).

On the other hand, let be given an $\{1, \cdots, d\}^{2}$-valued Markov chain $\left\{\left(Z_{n}, Y_{n}\right)\right\}_{n=1}^{\infty}$ on a probability space $\left(\mathbf{P}^{\prime}, \Omega^{\prime}\right)$. If it has the properties i) and ii) in Theorem (2.4), then following conclusions are obvious:

1) $p_{0}$ is a probability measure on $\{1, \cdots, d\}$ and $p_{0},\left(T_{j, i, k}\right)$ satisfy $(2.2 \mathrm{a}, \mathrm{b}, \mathrm{c})$;

2) the joint distribution of $\left\{Y_{n}\right\}_{n=1}^{\infty}$ is given by the right hand side of $(2.2 \mathrm{~d})$.

These end the proof.

Proof of Theorem (2.6). For any $m \in\{1, \cdots, d\}$, (3.5) shows that

$$
\mathscr{E}\left(\left|e_{m}><e_{m}\right| \otimes 1\right)=\sum_{r}\left|e_{r}><e_{r}\right| \operatorname{Tr}\left(\sum_{j} K_{j, m, r} K_{j, m, r}^{*}\right) .
$$

On the other hand, (2.5) makes sure that

$$
\mathscr{E}\left(\left|e_{m}><e_{m}\right| \otimes 1\right)=\left|e_{m}><e_{m}\right| .
$$

Comparing (3.23) and (3.24), we have

$$
\operatorname{Tr}\left(\sum_{j} K_{j, m, r} K_{j, m, r}^{*}\right)=\delta_{m, r}
$$

and this gives that

$$
K_{j, m, r}=0, \quad \forall m \neq r \in\{1,2, \cdots, d\}
$$

i.e. $K_{j}$ has the special form as (2.6). 
If any $K_{j}(j \in\{1, \cdots, d\})$ has the form.(2.6), we shall prove that

1) for each $a, b \in \mathscr{D}, \mathscr{E}(a \otimes b)=a \cdot \mathscr{E}(1 \otimes b)$;

2) for each $b \in \mathscr{D}, \mathscr{E}(1 \otimes b) \in \mathscr{D}$.

It is clear that with 1$)$, the condition 2$)$ is equivalent to

$2)^{\prime}$ for each $a, b \in \mathscr{D}, \mathscr{E}(a \otimes b) \in \mathscr{D}$.

The formula (3.5) shows, since any $K_{j}$ has the form (2.6), that

$$
\mathscr{E}(1 \otimes b)=\sum_{r}\left|e_{r}><e_{r}\right| \operatorname{Tr}\left(\sum_{j} K_{j, r, r}^{*} b K_{j, r, r}\right), \quad \forall b \in \mathscr{D}
$$

and this belongs to $\mathscr{D}$. Moreover

$$
\begin{gathered}
\mathscr{E}(a \otimes b)=\sum_{m} a(m, m)\left[\left|e_{m}><e_{m}\right| \operatorname{Tr}\left(\sum_{j} K_{j, m, m}^{*} b K_{j, m, m}\right)\right] \\
=\left(\begin{array}{cccc}
a(1,1) & 0 & \cdots & 0 \\
0 & a(2,2) & \cdots & 0 \\
\vdots & \vdots & \ddots & \vdots \\
0 & 0 & \cdots & a(d, d)
\end{array}\right) \\
\times\left(\begin{array}{cccc}
\operatorname{Tr}\left(\sum_{j} K_{j, 1,1}^{*} b K_{j, 1,1}\right) & 0 & \cdots & 0 \\
0 & \operatorname{Tr}\left(\sum_{j} K_{j, 2,2}^{*} b K_{j, 2,2}\right) & \cdots & 0 \\
\vdots & \vdots & \ddots & \vdots \\
0 & 0 & \cdots & \operatorname{Tr}\left(\sum_{j} K_{j, d, d}^{*} b K_{j, d, d}\right)
\end{array}\right) \\
=a \cdot \mathscr{E}(1 \otimes b) .
\end{gathered}
$$

This finishes the proof.

\section{REFERENCES}

[1] L. Accardi, On the noncommutative Markovian property, Funct. Anal. Appl., 9 (1975), 1-8.

[2] - Noncommutative Markov Chains, In: International School of Math. Phys. Camerino, (1974), 268-295.

[ 3 ] - Topics in quantum probability, Phy. Reports, 77, No. 3 (1981), 169-192.

[ 4 ] M. Fannes, B. Nachtergaele, R. F. Werner, Valence bond states on quantum spin chains as ground states with spectral gap, J. Phys. A: Math. Gen., 24 (1991), $185-190$.

Dipartimento di Matematica

Università di Bari

Centro V. Volterra

Università di Roma II
Current address:

Università degli Studi di Bari

Dipartimento di Matematica

Campus Universitario

Via E. Orabona, 4 